\title{
Evaluation of Inflammatory and Hematological Parameters in Patients Diagnosed with COVID-19
}

\author{
Çiğdem Mermutluoğlu ${ }^{1}$, Recep Dursun ${ }^{1}$, Fesih Aktar ${ }^{1}$, Saim Dayan ${ }^{1}$, Mustafa Kemal \\ Çelen ${ }^{1}$, Ali Kemal Kadiroğlu ${ }^{1}$, Erdal Inci ${ }^{1}$, Rengin Karagöz ${ }^{1}$, and Recep Tekin ${ }^{1}$ \\ ${ }^{1}$ Dicle University Medical Faculty
}

October 26, 2020

\begin{abstract}
Background: COVID-19, as a contagious infectious disease, can affect many systems and cause changes in inflammatory and hematological parameters. This study aims to research the effects of hematological and inflammatory parameters on prognosis of the disease and the duration of hospitalization. Methods: 186 patients aged 15-92 with positive COVID-19 PCR test results and a control group consisting of 187 healthy individuals with similar characteristics in terms of age and gender are included in the study. Age, gender, hematological variables, and inflammatory parameters of the patients on the 1st and 5th days of hospitalization were recorded retrospectively. Results: The mean age of the patient's group was $47.0 \pm 18.4$ years and $54.3 \%$ were male. WBC, lymphocyte count, and platelet count were statistically lower in the patient group compared to the control group, while MPV, NLR, and PLR levels were higher. On the 5th day of the patient follow-up compared to admission day, it was observed that the neutrophil count and MPV level were lower; However, the platelet count and ferritin level were statistically higher. While there was a significantly positive correlation between the duration of hospitalization and the 5 th day $\mathrm{D}$-dimer $(\mathrm{r}=$ 0546, $\mathrm{p}<0.001)$ and ferritin $(\mathrm{r}=0.568, \mathrm{p}<0.001)$; there was a negative correlation between the duration of hospitalization and admission day lymphocyte count $(\mathrm{r}=0.256, \mathrm{p}=0.006)$ and the 5 th day lymphocyte count $(\mathrm{r}=0.325, \mathrm{p}<0.001)$. Conclusion: Increased levels of ferritin and D-dimer and decreased count of lymphocyte are among important factors affecting the duration of hospitalization for COVID-19 patients. Furthermore, we think that neutrophil count and MPV levels are low and platelet count and ferritin levels are high during the course of the disease, so these parameters can be used as prognostic indicators of the disease.
\end{abstract}

\section{Introduction}

The coronavirus, which is accepted as a pandemic by the World Health Organization (WHO), as a member of the beta-coronavirus family, can affect all age groups and may appear with different clinical pictures (1). In the fight against this coronavirus (COVID-19) disease that causes a worldwide pandemic, the clinical and laboratory indicators of the progression towards severe and lethal forms need to be urgently identified. These predictors will enable risk classification, guide interventional efforts to target patients at high risk of developing severe disease and optimize the allocation of limited healthcare workers and technical resources in the ongoing pandemic. Moreover, defining the laboratory parameters that could be used to predict the clinical course of cases will allow an increase in clinical situational awareness. Therefore, monitoring of COVID-19 patients and effective early intervention are the most fundamental measures that can improve survival.

In line with the information obtained, the inflammatory response plays a critical role in the progression of COVID-19. It was reported that, on the one side, how the changes in acute phase reactants in COVID19 cases affect the course of the disease remains unclear, on the other side, it plays a significant role in disease severity and mortality. $(1,2)$. In some studies, hematological parameters are defined as potential indicators of disease severity $(3,4)$. However, to the best of our knowledge, there is no comprehensive study on hematological parameters, especially MPV, in adults who are infected with COVID-19. 
Hematological and inflammatory parameters such as white blood cell count (WBC), lymphocyte count, neutrophil count, platelet count, neutrophil to lymphocyte ratio (NLR), platelet to lymphocyte ratio (PLR), mean platelet volume (MPV), ferritin, D-dimer, fibrinogen, procalcitonin and C-reactive protein (CRP) in COVID-19 patients were defined as prognostic indicators of the disease (5-8).

We think that our findings will provide more information about the role of these indicators in determining the clinical course of patients diagnosed with COVID-19. Our aim in this study is to evaluate whether hematological and inflammatory biomarkers have prognostic effects and their effects on the duration of hospitalization in patients diagnosed with COVID-19 with PCR positivity and followed up by hospitalization.

\section{Material and Methods}

In this retrospective study between March and July 2020, 186 patients, who are aged between 15-92, hospitalized in Pandemic Hospital of Diyarbakir Dicle University, located in the east of Turkey, and diagnosed with COVID-19 and 187 persons, who show similarity with the patient group in terms of age and gender, were included in the control group. The medical information of the patients was obtained from the hospital records.

The diagnosis of COVID-19 was made using the real-time reverse transcriptase-polymerase chain reaction (RT-PCR) test based on the presence of a positive combined nasal/oral pharyngeal swab sample for SarsCoV-2 nucleic acid.

Age, gender, duration of hospitalization and WBC, neutrophil count, lymphocyte count, platelet count, MPV, NLR and PLR, ferritin, D-dimer, fibrinogen, procalcitonin, and CRP values of patients diagnosed with COVID-19 were collected retrospectively and recorded on the follow-up form on the 1st and 5th day of hospitalization. Also, age, gender, and hematological parameters (WBC, neutrophil count, lymphocyte count, platelet count, MPV, NLR, and PLR) of the control group were recorded.

NLR and PLR were calculated as the ratio of neutrophils to lymphocytes and the ratio of platelets to lymphocytes, respectively.

All blood samples taken for hematological parameters were tested for the same analyser, calibrated regularly.

Patients whose file records could not be reached were excluded from the study. Control groups were adults who were admitted to the hospital for routine control. Persons with any signs of infection or systemic disease and missing medical records were excluded from the control group.

This retrospective study protocol was approved by the Dicle University Faculty of Medicine Ethics Committee.

\section{Statistical Analysis}

Statistical analyses were performed using SPSS (version 19.0 program for Windows; SPSS Inc., Chicago, IL, USA). Categorical datas were presented as numbers (percentage) and tested using the Chi-square test or Fisher's exact test. Continuous variables with normal distribution were presented as mean \pm SD (standard deviation) and tested with a paired t-test. The chi-square test was used to compare categorical variables between the groups. Correlations between numerical variables were evaluated using Pearson's or Spearman's correlation analysis. We selected correlation strength by an absolute correlation $|r|>0.2$. P-values based on two-sided tests were considered as statistically significant at less than 0.05 .

\section{Results}

186 adults diagnosed with COVID-19 infection and 187 healthy control subjects were retrospectively evaluated. The mean age of the patient's group was $47.0 \pm 18.4$ years (15 - 92 years, min-max), and $54.3 \%$ $(\mathrm{n}=101)$ of the patients were male. The mean age of the control group was $44.0 \pm 12.7$ years (18 - 68 years, min-max) years, and $55.6 \%(\mathrm{n}=104)$ were male. There were no significant differences in the mean age $(\mathrm{p}=$ $0.067)$ or gender distribution $(\mathrm{p}=0.799)$ between the patient and the control subjects $(\mathrm{p}>0.05)$. The mean hospitalization duration was $7.2 \pm 4.1$ days (1-24 days, min-max) in the patient group. 
WBC $(\mathrm{p}=0.014)$, lymphocyte count $(\mathrm{p}<0.001)$ and platelet count $(\mathrm{p}=0.001)$ were statistically lower in the patient group compared to the control group, while MPV $(\mathrm{p}<0.001)$, NLR $(\mathrm{p}<0.001)$ and PLR $(\mathrm{p}<0.001)$ levels were higher. No significant difference was found between the two groups in terms of neutrophil count (Table 1).

Comparing the 1st and 5th-day laboratory parameters of the patients; it was found that neutrophil count (p $=0.035)$ and MPV $(\mathrm{p}=0.001)$ levels were found to be lower, while platelet count $(\mathrm{p}<0.001)$ and ferritin $(\mathrm{p}=0.026)$ level were found to be statistically higher on the 5th day of the follow-up compared to the time of admission. There was no statistically significant difference between day 1 and day 5 in terms of WBC, lymphocyte count, RDW, NLR, PLR, D-dimer, fibrinogen, CRP, and procalcitonin (Table 2).

While there was a moderately positive correlation between the duration of hospitalization and admission day neutrophil count $(\mathrm{r}=0.293, \mathrm{p}<0.001)$, NLR $(\mathrm{r}=0.273, \mathrm{p}=0.001)$, D-dimer $(\mathrm{r}=0.295, \mathrm{p}<0.001)$, CRP $(\mathrm{r}$ $=0.418, \mathrm{p}<0.001)$, procalcitonin $(\mathrm{r}=0.284, \mathrm{p}<0.001)$, fibrinogen $(\mathrm{r}=0.420, \mathrm{p}<0.001)$ and 5 th day NLR $(\mathrm{r}=0.413, \mathrm{p}<0.001)$, 5th day PLR $(\mathrm{r}=0.345, \mathrm{p}<0.001)$, 5th day CRP $(\mathrm{r}=0.480, \mathrm{p}<0.001)$, 5th day procalcitonin $(\mathrm{r}=0.387, \mathrm{p}=0.004)$, and 5 th day fibrinogen $(\mathrm{r}=0.301, \mathrm{p}<0.001)$ levels, there was a highly positive correlation between the 5th day D-dimer $(\mathrm{r}=0.546, \mathrm{p}<0.001)$ and the 5 th day ferritin $(\mathrm{r}=0.568, \mathrm{p}$ $<0.001)$. Moderately negative correlation was found between hospitalization duration and lymphocyte count at admission day $(\mathrm{r}=0.256, \mathrm{p}=0.006)$ and the 5th day lymphocyte count $(\mathrm{r}=0.325, \mathrm{p}<0.001)$.

\section{Discussion}

In this study, it was seen that low neutrophil count and MPV level, high platelet count and ferritin levels may be important monitoring parameters in the course and follow-up of COVID-19 infection. Besides, in our study, it was found that the laboratory levels of infection came up with important results affecting the duration of hospitalization.

As part of various studies on COVID-19 disease, it has been reported that the duration of hospitalization may be prolonged due to comorbidity conditions (obesity, diabetes, hypertension, and coronary heart disease), lymphopenia, high D-dimer and ferritin levels (11-13). However, studies on clinical and laboratory parameters that can determine the prognosis of patients are limited. As far as we know, there is no comprehensive study on prognostic factors affecting the duration of hospitalization in COVID-19 patients.

In our study, the mean hospitalization period of the patients was $7.2 \pm 4.1$ days. In correlation analysis between duration of hospitalization and laboratory parameters, a positive correlation was found between the duration of hospitalization and the 1st and 5th day NLR, CRP, procalcitonin, D-dimer, fibrinogen, the 5th day PLR, and ferritin levels. The low course of lymphocyte count both at admission and follow-up was seen to be the most important factor in prolonging the length of the duration of hospitalization. Therefore, it was observed that these parameters are factors affecting the duration of hospitalization in the follow-up of infection for COVID-19 patients in accordance with the literature.

It was reported as a result of a meta-analysis on COVID-19 patients that the male gender was predominant at a rate of $60 \%$, and the male gender at a rate of $63 \%$ with an average age of 46.4 was predominant in another study $(12,13)$. In our study, the mean age and male gender predominance of our patients were consistent with that of the literature.

As part of a study investigating the clinical characteristics of COVID-19 patients, $64.5 \%$ lymphopenia, and $29.4 \%$ leukopenia were found (12). In a study by Fu et al., $45.3 \%$ lymphopenia, $21.3 \%$ leukopenia, and $12 \%$ thrombocytopenia were found (7). In a study examining the laboratory parameters of 3377 patients, there was observed a slight increase in the WBC count in patients with severe disease, while there was a clinically significant increase in this parameter in patients who died (5). Therefore, in patients with severe disease, a significant increase in WBC may indicate clinical worsening and an increased risk of poor outcomes. Lymphopenia is a common feature in these patients, and a decrease is observed in the percentages of monocytes, eosinophils, and basophil cell percentages as well as CD4/CD8 T cells and natural killer cells $(1,5,14)$. It has been suggested that survival with COVID-19 may depend on its ability to regenerate lymphocytes killed by 
the virus. Therefore, the lymphocyte count, especially CD4, can be used as a clinical predictor of severity and prognosis (14). In our study, while the WBC and lymphocyte count were statistically lower in the patient group at the time of admission compared to the control group, no significant difference was found between the two groups in terms of neutrophil count. It was seen that neutrophil count was lower and there was no significant difference in the WBC and lymphocyte count on the 5th day of the follow-up of the patients compared to the time of admission. This indicates that COVID patients have lower WBC and lymphocyte count compared to the normal population; however, there is no significant change in the first 5 days of infection, and low levels of neutrophil should be closely monitored during the follow-up of the disease.

Increased neutrophil count and the associated increase in NLR levels are other findings observed in COVID-19 patients. It has been reported that the neutrophil to lymphocytes ratio (NLR), which can be easily calculated from a routine blood test by dividing the absolute neutrophil count to the absolute number of lymphocytes, has a significant value in demonstrating the general inflammatory status of a patient (6). In a study, it was found that neutrophil rate was $51.6 \%$ and the increased NLR level was associated with the severity of the disease, poor prognosis, and duration of hospitalization (8). In our study, while the NLR level was higher in the patient group compared to the control group at the time of admission, there was no significant difference in the 1st and 5th day NLR levels of the patients. In light of these results, we believe that the NLR level may be important in the diagnosis of the COVID-19 infection; however, it has no prognostic significance in the follow-up of the disease.

Thrombocytes are immune cells that play an important role in the human body. The incidence of thrombocytopenia in critically ill patients admitted to the intensive care unit varies between $15-60 \%$ (5). In a meta-analysis where the relationship between thrombocytopenia and COVID-19 severity is investigated, low platelet count was found to increase the risk of severe disease and mortality three times in COVID-19 patients and prolonged the length of hospitalization (3). According to another study, platelet peaks and increased PLR levels observed in the follow-up of symptomatic and asymptomatic patients were found to be associated with the severity of the disease and the duration of hospitalization (8). The PLR level is calculated by dividing the absolute platelet count by the absolute lymphocyte count. The high level of PLR of the patients refers to the degree of cytokine storm that can provide a new indicator in the follow-up of patients with COVID-19 $(8,15)$.

Mean platelet volume (MPV) is a laboratory marker associated with platelet function and activity. High MPV is hemostatically more reactive and produces higher amounts of the prothrombotic factor, thromboxane. This increased thromboxane causes thrombotic sensitivity and therefore results in thrombotic complications (16). According to a study by Elsayed and Mohamed, it was reported that increasing MPV and MPV/thrombocyte count ratio is an important risk factor in the development of thromboembolic events (17). In the study by Pan et al., the increased MPV level was found to be a distinctive feature in COVID-19 patients (18). In our study, the number of platelets at the time of admission was significantly lower, while PLR and MPV levels were higher in the patient group compared to the control group. It was found that the MPV level was lower and the platelet count was higher on the 5th day of the patient follow-up compared to the time of admission. We think that these results may be associated with platelet levels increased by the improvement of thrombocytopenia with the healing process in patients with initial thrombocytopenia or by the progress of the disease in patients with normal thrombocyte levels at the beginning. However, due to the lack of sufficient and high level of evidence about this situation, we believe that studies with a larger patient series should be carried out.

We think that the high MPV level in the early stage of the disease and its low course in the follow-up may be related to the prognosis. Therefore, the MPV level can be used as a good prognostic factor in the follow-up of COVID-19 infection.

Ferritin that is used as an inflammation marker significantly increased during the course of the disease in patients with severe COVID-19. Therefore, serum ferritin levels should be used to monitor prognosis in COVID-19 patients during hospitalization. The increase in the CRP level with ferritin indicates the development of systemic inflammatory response syndrome. The exaggerated elevation that may lead to 
cytokine storm can lead to tissue damage progressing to acute lung injury and multi-organ failure $(19,20)$. In a study examining the relationship between the severity of the COVID-19 disease and procalcitonin, a significant difference was observed only in the procalcitonin level between severe and non-severe forms of the disease. However, in another study, an approximately five-fold higher risk of severe COVID-19 infection was observed in patients with increased procalcitonin $(4,20)$. D-dimer occurs due to the destruction of the fibrin clot formed by cross-links by the plasmin with the activation of the coagulation system for any reason (5). In a study by Zhang et al., comparing D-dimer levels at the time of admission, it was stated that a high D-dimer level at the time of admission was an important factor affecting mortality (21). D-dimer is significantly elevated in patients with both severe and fatal COVID-19 (5). In our study, significantly high levels of ferritin were observed on the 5th day compared to the time of admission. However, no significant difference was observed in CRP, procalcitonin, D-dimer, and fibrinogen levels in prognostic terms. With these results, we think that, instead of inflammatory markers such as CRP and procalcitonin and thrombosis indicators such as D-dimer, the ferritin level should be closely monitored to predict the severity of the infection.

Our study had limitations such as being retrospective performed in a single center and a short study period, and not investigating other inflammatory indicators such as lymphocyte subtypes and cytokines.

\section{Conclusion}

Consequently, the SARS-CoV-2 virus is spreading by leaps and bounds all over the world. The prognosis of the disease may vary depending on the nature of a person's immune system. In this study, where hematological and inflammatory characteristics of peripheral blood were analysed, it has been shown that increased ferritin and D-dimer levels and decreased lymphocyte count are important factors affecting the duration of hospitalization for COVID-19 infection. Moreover, we point out low neutrophil count and MPV level and high platelet count and ferritin levels during the course of the disease, so these parameters can be used as prognostic indicators of the disease.

\section{References}

1. Mehta P, McAuley DF, Brown M, Sanchez E, Tattersall RS, Manson JJ, et al. COVID 19: consider cytokine storm syndromes and immunosuppression. Lancet 2020;395(10229):1033-4.

2. Stebbing J, Phelan A, Griffin I, Tucker C, Oechsle O, Smith D, et al. COVID-19: combining antiviral and anti-inflammatory treatments. Lancet Infect Dis 2020;20(4):400-2.

3. Lippi G, Plebani M, Henry BM. Thrombocytopenia is associated with severe coronavirus disease 2019 (COVID-19) infections: a meta-analysis. Clin Chim Acta 2020. doi: https://doi.org/10.1016/j.cca.2020.03.022.

4. Lippi G, Plebani M. Procalcitonin in patients with severe coronavirus disease 2019 (COVID-19): a meta-analysis. Clin Chim Acta 2020. doi: 10.1016/j.cca.2020.03.004.

5. Henry BM, de Oliveira MHS, Benoit S, Plebani M, Lippi G. Hematologic, biochemical and immune biomarker abnormalities associated with severe illness and mortality in coronavirus disease 2019 (COVID19): a meta-analysis. Clin Chem Lab Med 2020;58(7):1021-1028. doi: 10.1515/cclm-2020-0369.

6. Lagunas-Rangel FA. Neutrophil-to-lymphocyte ratio and lymphocyte-to-C-reactive protein ratio in patients with severe coronavirus disease 2019 (COVID-19): A meta-analysis. J Med Virol 2020:10.1002/jmv.25819. doi: 10.1002/jmv.25819.

7. Fu J, Kong J, Wang W, Wu M, Yao L, Wang Z, et al. The clinical implication of dynamic neutrophil to lymphocyte ratio and D-dimer in COVID-19: A retrospective study in Suzhou China. Thromb Res 2020;192:3-8. doi: 10.1016/j.thromres.2020.05.006.

8. Yang AP, Liu JP, Tao WQ, Li HM. The diagnostic and predictive role of NLR, d-NLR and PLR in COVID-19 patients. Int Immunopharmacol 2020;84:106504. doi: 10.1016/j.intimp.2020.106504.

9. Du RH, Liu LM, Yin W, Wang W, Guan LL, Yuan ML, et al. Hospitalization and critical care of 109 decedents with COVID-19 pneumonia in Wuhan, China. Ann Am Thorac Soc 2020;17(7):839-846. doi: 10.1513/AnnalsATS.202003-225OC.

10. Li H, Liu SM, Yu XH, Tang SL, Tang CK. Coronavirus disease 2019 (COVID-19): current status and 
future perspectives. Int J Antimicrob Agents 2020;55(5):105951. doi: 10.1016/j.ijantimicag.2020.105951.

11. Guan WJ, Liang WH, Zhao Y, Liang HR, Chen ZS, Li YM, et al. Comorbidity and its impact on 1590 patients with COVID-19 in China: a nationwide analysis. Eur Respir J 2020;55(5):2000547. doi: 10.1183/13993003.00547-2020.

12. Li LQ, Huang T, Wang YQ, Wang ZP, Liang Y, Huang TB, et al. COVID-19 patients' clinical characteristics, discharge rate, and fatality rate of meta-analysis. J Med Virol 2020;92(6):577-583. doi: 10.1002/jmv.25757.

13. Yang AP, Liu JP, Tao WQ, Li HM. The diagnostic and predictive role of NLR, d-NLR and PLR in COVID-19 patients. Int Immunopharmacol 2020;84:106504. doi: 10.1016/j.intimp.2020.106504.

14. Henry BM. COVID-19, ECMO, and lymphopenia: a word of caution. Lancet Respir Med 2020;8(4):e24. doi: 10.1016/S2213-2600(20)30119-3.

15. Qu R, Ling Y, Zhang Y, Wei L, Chen X, Xu-Li M, et al. Platelet-to-lymphocyte ratio is associated with prognosis in patients with coronavirus disease-19. J Med Virol 2020. doi: 10.1002/jmv.25767.

16. Ozder A. A novel indicator predicts 2019 novel coronavirus infection in subjects with diabetes. Diabetes Res Clin Pract 2020;166:108294. doi:10.1016/j.diabres.2020.108294

17. Elsayed AM, Mohamed GA. Mean platelet volume and mean platelet volume/platelet count ratio as a risk stratification tool in the assessment of severity of acute ischemic stroke. Alexandria Journal of Medicine 2017;53(1).doi.org/10.1016/j.ajme.2016.03.003

18. Pan Y, Ye G, Zeng X, Liu G, Zeng X, Jiang X, et al. Can routine laboratory tests discriminate SARSCoV-2-infected pneumonia from other causes of community-acquired pneumonia?. Clin Transl Med 2020;10(1):161-168. doi: 10.1002/ctm2.23.

19. Huang I, Pranata R, Lim MA, Oehadian A, Alisjahbana B. C-reactive protein, procalcitonin, D-dimer, and ferritin in severe coronavirus disease-2019: a meta-analysis. Ther Adv Respir Dis 2020;14:1753466620937175. doi: 10.1177/1753466620937175.

20. Terpos E, Ntanasis-Stathopoulos I, Elalamy I, Kastritis E, Sergentanis TN, Politou M, et al. Hematological findings and complications of COVID-19. Am J Hematol 2020;95(7):834-847. doi:10.1002/ajh.25829.

21. Zhang L, Yan X, Fan Q, Liu H, Liu X, Liu Z, et al. D-dimer levels on admission to predict in-hospital mortality in patients with Covid-19. J Thromb Haemost 2020;18(6):1324-1329. doi:10.1111/jth.14859.

\section{Conflict of Interest}

All authors declare that there is no conflict of interest.

\section{Funding}

None

\section{Authorship}

FA and ÇM and SD made the conception and design of the study, wrote the manuscript and approved the final version to be submitted. RD, EA and RK involved drafting the article and interpretation of data, made acquisition of data. MKÇ, AKK, and RT revised manuscript critically for important intellectual content. All authors agreed to be accountable for all aspects of the work in ensuring that questions related to the accuracy or integrity of any part of the work are appropriately investigated and resolved. All authors read and approved the final version of the manuscript.

\section{Hosted file}

Table 1.pdf available at https://authorea.com/users/341450/articles/488766-evaluation-ofinflammatory-and-hematological-parameters-in-patients-diagnosed-with-covid-19

\section{Hosted file}

Table 2.pdf available at https://authorea.com/users/341450/articles/488766-evaluation-ofinflammatory-and-hematological-parameters-in-patients-diagnosed-with-covid- 19 